\title{
SOME PROPERTIES OF DIFFERENTIAL OPERATOR ASSOCIATED WITH GENERALIZED HYPERGEOMETRIC FUNCTIONS
}

\author{
ENTISAR EL-YAGUBI AND MASLINA DARUS
}

\begin{abstract}
In the present investigation, new subclasses of analytic functions in the open unit disk which are defined using generalized derivative operator are introduced. Several interesting properties of these classes are obtained.
\end{abstract}

\section{Introduction}

Let $\mathscr{A}$ denote the class of functions of the form

$$
f(z)=z+\sum_{n=2}^{\infty} a_{n} z^{n}
$$

which are analytic in the open unit disk $\mathbb{U}=\{z: z \in \mathbb{C},|z|<1\}$. Also let $\mathscr{S}$ be the the subclass of $\mathscr{A}$ consisting of all functions which are univalent in $\mathbb{U}$. If $f(z) \in \mathscr{A}$ satisfies

$$
\left|\arg \left(\frac{z f^{\prime}(z)}{f(z)}-\alpha\right)\right|<\frac{\pi}{2} \beta,(z \in U, 0 \leq \alpha<1,0<\beta \leq 1),
$$

then $f(z)$ is said to be strongly starlike of order $\beta$ and type $\alpha$ in $\mathbb{U}$, and denoted by $S^{*}(\alpha, \beta)$. If $f(z) \in \mathscr{A}$ satisfies

$$
\left|\arg \left(1+\frac{z f^{\prime \prime}(z)}{f^{\prime}(z)}-\alpha\right)\right|<\frac{\pi}{2} \beta,(z \in U, 0 \leq \alpha<1,0<\beta \leq 1),
$$

then $f(z)$ is said to be strongly convex of order $\beta$ and type $\alpha$ in $\mathbb{U}$, and denoted by $C(\alpha, \beta)$.

It is obvious that $f(z) \in \mathscr{A}$ belongs to $C(\alpha, \beta)$ if and only if $z f^{\prime}(z) \in S^{*}(\alpha, \beta)$. Further, we note that $S^{*}(\alpha, 1) \equiv S^{*}(\alpha)$ and $C(\alpha, 1) \equiv C(\alpha)$ which are, respectively, starlike and convex univalent functions of order $\alpha$. Let $\mathscr{P}$ denote the class of functions of the form $p(z)=1+$ $p_{1}(z)+\cdots$ analytic in $\mathbb{U}$ which satisfy the condition $\Re\{p(z)\}>0$.

Received January 26, 2014, accepted June 10, 2014.

2010 Mathematics Subject Classification. 30C45.

Key words and phrases. Analytic functions, differential operator, starlike functions, convex functions, subordination, linear operator.

Corresponding author: Entisar El-Yagubi. 
For two functions given by $f(z)=\sum_{n=2}^{\infty} a_{n} z^{n}$ and $g(z)=\sum_{n=2}^{\infty} b_{n} z^{n}$ be analytic in $\mathbb{U}$. Then the Hadamard product (or convolution) $f * g$ of the two functions $f, g$ is defined by

$$
f(z) * g(z)=z+\sum_{n=2}^{\infty} a_{n} b_{n} z^{n} .
$$

Recall that the function $F$ is subordinate to $G$ if there exists a function $\omega$, analytic in $\mathbb{U}$, with $\omega(0)=0$ and $|\omega(z)|<1$ such that $F(z)=G(\omega(z)), z \in \mathbb{U}$. We denote this subordination by $F(z)<$ $G(z)$. If $G(z)$ is univalent in $\mathbb{U}$, then the subordination is equivalent to $F(0)=G(0)$ and $F(\mathbb{U}) \subset$ $G(\mathbb{U})$.

For complex parameters $a_{i}, b_{j},\left(i=1, \ldots, r, j=1, \ldots, s, b_{j} \in \mathbb{C} \backslash\{0,-1,-2, \ldots\}\right)$, we shall use the generalized hypergeometric function ${ }_{r} \Phi_{s}\left(a_{i}, b_{j} ; z\right)$

$$
{ }_{r} \Phi_{s}\left(a_{i}, b_{j} ; z\right)=\sum_{n=0}^{\infty} \frac{\left(a_{1}\right)_{n} \cdots\left(a_{r}\right)_{n}}{\left(b_{1}\right)_{n} \cdots\left(b_{s}\right)_{n}} \frac{z^{n}}{n !},
$$

where $r \leq s+1 ; r, s \in \mathbb{N}_{0}=\mathbb{N} \cup\{0\} ; z \in \mathbb{U}, \mathbb{N}$ denotes the set of positive integers and $(x)_{n}$ is the Pochhammer symbol defined in terms of the Gamma function $\Gamma$, by

$$
(x)_{n}=\frac{\Gamma(x+n)}{\Gamma(x)}= \begin{cases}1, & n=0, \\ x(x+1) \cdots(x+n-1), & n=\{1,2,3, \ldots\} .\end{cases}
$$

Corresponding to a function ${ }_{r} \mathscr{G}_{s}\left(a_{i}, b_{j} ; z\right)$ defined by

$$
{ }_{r} \mathscr{G}_{s}\left(a_{i}, b_{j} ; z\right)=z_{r} \Phi_{s}\left(a_{i}, b_{j} ; z\right)
$$

Dziok and Srivastava [1] introduced a convolution operator on $\mathscr{A}$ such that

$$
\mathscr{H}_{r, s}\left(a_{i}, b_{j}\right): \mathscr{A} \rightarrow \mathscr{A}
$$

is defined by

$$
\begin{aligned}
\mathscr{H}_{r, s}\left(a_{i}, b_{j}\right) f(z) & ={ }_{r} \mathscr{G}_{s}\left(a_{i}, b_{j} ; z\right) * f(z) \\
& =z+\sum_{n=2}^{\infty} \frac{\left(a_{1}\right)_{n-1} \cdots\left(a_{r}\right)_{n-1}}{\left(b_{1}\right)_{n-1} \cdots\left(b_{s}\right)_{n-1}} \frac{a_{n} z^{n}}{(n-1) !} .
\end{aligned}
$$

We now define the following operator $\mathscr{D}_{\lambda_{1}, \lambda_{2}}^{m, b}\left(a_{i}, b_{j}\right) f: \mathscr{A} \rightarrow \mathscr{A}$ by the following Hadamard product:

$$
\begin{aligned}
\mathscr{D}_{\lambda_{1}, \lambda_{2}}^{0, b}\left(a_{i}, b_{j}\right) f(z)= & { }_{r} \mathscr{G}_{S}\left(a_{i}, b_{j} ; z\right) * f(z), \\
(1+b) \mathscr{D}_{\lambda_{1}, \lambda_{2}}^{1, b}\left(a_{i}, b_{j}\right) f(z)= & \left(1-\left(\lambda_{1}+\lambda_{2}\right)+b\right)\left(\varphi_{\lambda_{2}}^{b} *_{r} \mathscr{G}_{s}\left(a_{i}, b_{j} ; z\right) * f(z)\right) \\
& +\left(\lambda_{1}+\lambda_{2}\right) z\left(\varphi_{\lambda_{2}}^{b} *_{r} \mathscr{G}_{s}\left(a_{i}, b_{j} ; z\right) * f(z)\right)^{\prime},
\end{aligned}
$$




$$
\mathscr{D}_{\lambda_{1}, \lambda_{2}}^{m, b}\left(a_{i}, b_{j}\right) f(z)=\mathscr{D}_{\lambda_{1}, \lambda_{2}}^{b}\left(\mathscr{D}_{\lambda_{1}, \lambda_{2}}^{m-1, b}\left(a_{i}, b_{j}\right) f(z)\right),
$$

where $\varphi_{\lambda_{2}}^{b}=z+\sum_{n=2}^{\infty} \frac{z^{n}}{1+\lambda_{2}(n-1)+b}$.

From (1.5) and (1.6) we may easily deduce the following linear operator:

$$
\mathscr{D}_{\lambda_{1}, \lambda_{2}}^{m, b}\left(a_{i}, b_{j}\right) f(z)=z+\sum_{n=2}^{\infty}\left[\frac{1+\left(\lambda_{1}+\lambda_{2}\right)(n-1)+b}{1+\lambda_{2}(n-1)+b}\right]^{m} \frac{\left(a_{1}\right)_{n-1} \cdots\left(a_{r}\right)_{n-1}}{\left(b_{1}\right)_{n-1} \cdots\left(b_{s}\right)_{n-1}} \frac{a_{n} z^{n}}{(n-1) !},
$$

where $\left.\lambda_{2} \geq \lambda_{1} \geq 0, m, b \in \mathbb{N}_{0}=\{0,1,2, \ldots\}, a_{i} \in \mathbb{C}, b_{j} \in \mathbb{C} \backslash\{0,-1,-2, \ldots\}\right)$, $\left(i=1, \ldots, r, j=1, \ldots, s\right.$, and $r \leq s+1 ; r, s \in \mathbb{N}_{0}$.

It should be remarked that the linear operator (1.7) is a generalization of many operators considered earlier. Let us see some of the examples:

For $m=0$ the operator $\mathscr{D}_{\lambda_{1}, \lambda_{2}}^{m, b}\left(a_{i}, b_{j}\right) f$ reduces to the well-known Dziok- Srivastava operator [1]. For $\lambda_{2}=b=0$, we get the Selvaraj derivative operator [2]. For $m=0, r=2$ and $s=1$ we obtain the Hohlov derivative operator [3]. For $r=1, s=0, a_{1}=1, \lambda_{1}=1$ and $\lambda_{2}=b=0$, we get the Salagean derivative operator [4]. For $r=1, s=0, a_{1}=1$ and $\lambda_{2}=b=0$, we get the generalized Salagean derivative operator introduced by Al-Oboudi [5]. For $m=0, r=1, s=0$ and $a_{1}=\delta+1$ we obtain the Ruscheweyh derivative operator [6]. For $r=1, s=0$ and $a_{1}=\delta+1$ we obtain the derivative given by El-Yagubi and Darus [7]. For $m=0, r=2$ and $s=1$ and $a_{2}=1$ we obtain the Carlson and Shaffer [8]. For $r=1, s=0, a_{1}=1$ and $\lambda_{2}=0$ we get the Cátás derivative operator [9].

Remark 1.1. It follows from the above definition that:

$$
\begin{aligned}
(1+b) \mathscr{D}_{\lambda_{1}, \lambda_{2}}^{m+1, b}\left(a_{i}, b_{j}\right) f(z)= & \left(1-\left(\lambda_{1}+\lambda_{2}\right)+b\right)\left(\mathscr{D}_{\lambda_{1}, \lambda_{2}}^{m, b}\left(a_{i}, b_{j}\right) * \varphi_{\lambda_{2}}^{b} f(z)\right) \\
& +\left(\lambda_{1}+\lambda_{2}\right) z\left(\mathscr{D}_{\lambda_{1}, \lambda_{2}}^{m, b}\left(a_{i}, b_{j}\right) * \varphi_{\lambda_{2}}^{b} f(z)\right)^{\prime},
\end{aligned}
$$

and

$$
a_{1} \mathscr{D}_{\lambda_{1}, \lambda_{2}}^{m, b}\left(a_{1}+1, b_{j}\right) f(z)=\left(a_{1}-1\right) \mathscr{D}_{\lambda_{1}, \lambda_{2}}^{m, b}\left(a_{1}, b_{j}\right) f(z)+z\left(\mathscr{D}_{\lambda_{1}, \lambda_{2}}^{m, b}\left(a_{1}, b_{j}\right) f(z)\right)^{\prime}
$$

Now, we introduce the following classes in terms of the new operator $\mathscr{D}_{\lambda_{1}, \lambda_{2}}^{m, b}\left(a_{i}, b_{j}\right)$ :

Definition 1.1. For $\left.\lambda_{2} \geq \lambda_{1} \geq 0, m, b \in \mathbb{N}_{0}=\{0,1,2, \ldots\}, a_{i} \in \mathbb{C}, b_{j} \in \mathbb{C} \backslash\{0,-1,-2, \ldots\}\right),(i=$ $1, \ldots, r, j=1, \ldots, s, r \leq s+1 ; r, s \in \mathbb{N}_{0}, 0 \leq \alpha<1$ and $0<\beta \leq 1$, let $\mathscr{S}_{\lambda_{1}, \lambda_{2}}^{m, b}\left(a_{i}, b_{j} ; \alpha, \beta\right)$ be the class of functions $f \in \mathscr{A}$ satisfying

$$
\left|\arg \left(\frac{z\left(\mathscr{D}_{\lambda_{1}, \lambda_{2}}^{m, b}\left(a_{i}, b_{j}\right) f(z)\right)^{\prime}}{\mathscr{D}_{\lambda_{1}, \lambda_{2}}^{m, b}\left(a_{i}, b_{j}\right) f(z)}-\alpha\right)\right|<\frac{\pi}{2} \beta \quad(z \in \mathbb{U}),
$$

where $\mathscr{D}_{\lambda_{1}, \lambda_{2}}^{m, b}\left(a_{i}, b_{j}\right) f(z) \in S^{*}(\alpha, \beta)$ and $\frac{z\left(\mathscr{D}_{\lambda_{1}, \lambda_{2}}^{m, b}\left(a_{i}, b_{j}\right) f(z)\right)^{\prime}}{\mathscr{D}_{\lambda_{1}, \lambda_{2}}^{m, b}\left(a_{i}, b_{j}\right) f(z)} \neq \alpha$. 
Definition 1.2. For $\left.\lambda_{2} \geq \lambda_{1} \geq 0, m, b \in \mathbb{N}_{0}=\{0,1,2, \ldots\}, a_{i} \in \mathbb{C}, b_{j} \in \mathbb{C} \backslash\{0,-1,-2, \ldots\}\right),(i=$ $1, \ldots, r, j=1, \ldots, s, r \leq s+1 ; r, s \in \mathbb{N}_{0}, 0 \leq \alpha<1$ and $0<\beta \leq 1$, let $\mathscr{C}_{\lambda_{1}, \lambda_{2}}^{m, b}\left(a_{i}, b_{j} ; \alpha, \beta\right)$ be the class of functions $f \in \mathscr{A}$ satisfying

$$
\left|\arg \left(1+\frac{z\left(\mathscr{D}_{\lambda_{1}, \lambda_{2}}^{m, b}\left(a_{i}, b_{j}\right) f(z)\right)^{\prime \prime}}{\left(\mathscr{D}_{\lambda_{1}, \lambda_{2}}^{m, b}\left(a_{i}, b_{j}\right) f(z)\right)^{\prime}}-\alpha\right)\right|<\frac{\pi}{2} \beta \quad(z \in \mathbb{U}),
$$

where $\mathscr{D}_{\lambda_{1}, \lambda_{2}}^{m, b}\left(a_{i}, b_{j}\right) f(z) \in C(\alpha, \beta)$ and $1+\frac{z\left(\mathscr{D}_{\lambda_{1}, \lambda_{2}}^{m, b}\left(a_{i}, b_{j}\right) f(z)\right)^{\prime \prime}}{\left(\mathscr{D}_{\lambda_{1}, \lambda_{2}}^{m, b}\left(a_{i}, b_{j}\right) f(z)\right)^{\prime}} \neq \alpha$.

Clearly, $f \in \mathscr{C}_{\lambda_{1}, \lambda_{2}}^{m, b}\left(a_{i}, b_{j} ; \alpha, \beta\right)$ if and only if $z f^{\prime}(z) \in \mathscr{S}_{\lambda_{1}, \lambda_{2}}^{m, b}\left(a_{i}, b_{j} ; \alpha, \beta\right)$.

Note that $\mathscr{S}_{\lambda_{1}, \lambda_{2}}^{0, b}(1,1 ; \alpha, \beta) \equiv S^{*}(\alpha, \beta), \mathscr{S}_{\lambda_{1}, \lambda_{2}}^{0, b}(1,1 ; \alpha, 1) \equiv S^{*}(\alpha), \mathscr{C}_{\lambda_{1}, \lambda_{2}}^{0, b}(1,1 ; \alpha, \beta) \equiv C(\alpha, \beta)$ and $\mathscr{C}_{\lambda_{1}, \lambda_{2}}^{0, b}(1,1 ; \alpha, 1) \equiv C(\alpha)$.

Definition 1.3. For $\left.\lambda_{2} \geq \lambda_{1} \geq 0, m, b \in \mathbb{N}_{0}=\{0,1,2, \ldots\}, a_{i} \in \mathbb{C}, b_{j} \in \mathbb{C} \backslash\{0,-1,-2, \ldots\}\right),(i=$ $1, \ldots, r, j=1, \ldots, s, r \leq s+1 ; r, s \in \mathbb{N}_{0}, 0 \leq \gamma<1$ and $-1 \leq B<A \leq 1$, let $\mathscr{Q}_{\lambda_{1}, \lambda_{2}}^{m, b}\left(a_{i}, b_{j} ; \gamma ; A, B\right)$ be the class of functions $f \in \mathscr{A}$ satisfying

$$
\frac{1}{1-\gamma}\left(\frac{z\left(\mathscr{D}_{\lambda_{1}, \lambda_{2}}^{m, b}\left(a_{i}, b_{j}\right) f(z)\right)^{\prime}}{\mathscr{D}_{\lambda_{1}, \lambda_{2}}^{m, b}\left(a_{i}, b_{j}\right) f(z)}-\gamma\right) \prec \frac{1+A z}{1+B z} \quad(z \in \mathbb{U}),
$$

where $\mathscr{D}_{\lambda_{1}, \lambda_{2}}^{m, b}\left(a_{i}, b_{j}\right) f(z) \in S^{*}(\gamma ; A, B)$ and $\frac{z\left(\mathscr{D}_{\lambda_{1}, \lambda_{2}}^{m, b}\left(a_{i}, b_{j}\right) f(z)\right)^{\prime}}{\mathscr{D}_{\lambda_{1}, \lambda_{2}}^{m, b}\left(a_{i}, b_{j}\right) f(z)} \neq \gamma$.

\section{Main results}

To derive our theorems, the following lemma will be required.

Lemma 2.1 (see [10]). Let $\beta, v$ be complex numbers. Let $\phi \in \mathscr{P}$ be convex univalent in $\mathbb{U}$ with $\phi(0)=1$ and $\Re\{\beta \phi(z)+v\}>0, z \in \mathbb{U}$. If $p(z)=1+p_{1} z+p_{2} z^{2}+\cdots$ is analytic in $\mathbb{U}$ with $p(0)=1$, then

$$
p(z)+\frac{z p^{\prime}(z)}{\beta p(z)+v} \prec \phi(z) \Rightarrow p(z) \prec \phi(z), \quad(z \in \mathbb{U}) .
$$

Theorem 2.2. Let $\phi(z)$ be convex univalent in $\mathbb{U}$ with $\phi(0)=1$ and $\Re\{\phi(z)\} \geq 0$. Iff $\in \mathscr{A}$ satisfies the condition

$$
\frac{1}{1-\gamma}\left(\frac{z\left(\mathscr{D}_{\lambda_{1}, \lambda_{2}}^{m+1, b}\left(a_{i}, b_{j}\right) f(z)\right)^{\prime}}{\mathscr{D}_{\lambda_{1}, \lambda_{2}}^{m+1, b}\left(\alpha_{i}, \beta_{j}\right) f(z)}-\gamma\right) \prec \phi(z) \quad(z \in \mathbb{U})
$$

then 


$$
\frac{1}{1-\gamma}\left(\frac{z\left(\mathscr{D}_{\lambda_{1}, \lambda_{2}}^{m, b}\left(\alpha_{i}, \beta_{j}\right) * \varphi_{\lambda_{2}}^{b} f(z)\right)^{\prime}}{\mathscr{D}_{\lambda_{1}, \lambda_{2}}^{m, b}\left(\alpha_{i}, \beta_{j}\right) * \varphi_{\lambda_{2}}^{b} f(z)}-\gamma\right)<\phi(z) \quad(z \in \mathbb{U}),
$$

for $\left.\lambda_{2} \geq \lambda_{1} \geq 0, m, b \in \mathbb{N}_{0}, a_{i} \in \mathbb{C}, b_{j} \in \mathbb{C} \backslash\{0,-1,-2, \ldots\}\right),\left(i=1, \ldots, r, j=1, \ldots, s, r \leq s+1 ; r, s \in \mathbb{N}_{0}\right.$ and $0 \leq \gamma<1$.

Proof. Let

$$
p(z)=\frac{1}{1-\gamma}\left(\frac{z\left(\mathscr{D}_{\lambda_{1}, \lambda_{2}}^{m, b}\left(a_{i}, b_{j}\right) * \varphi_{\lambda_{2}}^{b} f(z)\right)^{\prime}}{\mathscr{D}_{\lambda_{1}, \lambda_{2}}^{m, b}\left(a_{i}, b_{j}\right) * \varphi_{\lambda_{2}}^{b} f(z)}-\gamma\right),
$$

where $p \in \mathscr{P}$. By using equation (1.8) in (2.1) and differentiating logarithmically, we get

$$
\begin{aligned}
& \frac{1}{1-\gamma}\left(\frac{z\left(\mathscr{D}_{\lambda_{1}, \lambda_{2}}^{m+1, b}\left(a_{i}, b_{j}\right) f(z)\right)^{\prime}}{\mathscr{D}_{\lambda_{1}, \lambda_{2}}^{m+1, b}\left(a_{i}, b_{j}\right) f(z)}-\gamma\right) \\
& \quad=p(z)+\frac{z p^{\prime}(z)}{(1-\gamma) p(z)+\left(\left(1-\left(\lambda_{1}+\lambda_{2}\right)+b\right)+\gamma\right) /\left(\lambda_{1}+\lambda_{2}\right)} .
\end{aligned}
$$

Since $\frac{1}{1-\gamma}\left(\frac{z\left(\mathscr{D}_{\lambda_{1}, \lambda_{2}}^{m+1, b}\left(a_{i}, b_{j}\right) f(z)\right)^{\prime}}{\mathscr{D}_{\lambda_{1}, \lambda_{2}}^{m+1, b}\left(\alpha_{i}, \beta_{j}\right) f(z)}-\gamma\right) \prec \phi(z)$, and applying Lemma 2.1, it follows that $p<\phi$. Hence the required result is obtained.

Theorem 2.3. Let $\phi(z)$ be convex univalent in $\mathbb{U}$ with $\phi(0)=1$ and $\Re\{\phi(z)\} \geq 0$. Iff $\in \mathscr{A}$ satisfies the condition

$$
\frac{1}{1-\gamma}\left(\frac{z\left(\mathscr{D}_{\lambda_{1}, \lambda_{2}}^{m, b}\left(a_{1}+1, b_{j}\right) f(z)\right)^{\prime}}{\mathscr{D}_{\lambda_{1}, \lambda_{2}}^{m, b}\left(a_{1}+1, b_{j}\right) f(z)}-\gamma\right) \prec \phi(z) \quad(z \in \mathbb{U})
$$

then

$$
\frac{1}{1-\gamma}\left(\frac{z\left(\mathscr{D}_{\lambda_{1}, \lambda_{2}}^{m, b}\left(a_{i}, b_{j}\right) f(z)\right)^{\prime}}{\mathscr{D}_{\lambda_{1}, \lambda_{2}}^{m, b}\left(a_{i}, b_{j}\right) f(z)}-\gamma\right)<\phi(z) \quad(z \in \mathbb{U}),
$$

for $\left.\lambda_{2} \geq \lambda_{1} \geq 0, m, b \in \mathbb{N}_{0}=\{0,1,2, \ldots\}, a_{i} \in \mathbb{C}, b_{j} \in \mathbb{C} \backslash\{0,-1,-2, \ldots\}\right),(i=1, \ldots, r, j=1, \ldots, s, r \leq$ $s+1 ; r, s \in \mathbb{N}_{0}$ and $0 \leq \gamma<1$.

Proof. Let

$$
p(z)=\frac{1}{1-\gamma}\left(\frac{z\left(\mathscr{D}_{\lambda_{1}, \lambda_{2}}^{m, b}\left(a_{i}, b_{j}\right) f(z)\right)^{\prime}}{\mathscr{D}_{\lambda_{1}, \lambda_{2}}^{m, b}\left(a_{i}, b_{j}\right) f(z)}-\gamma\right),
$$

where $p \in \mathscr{P}$. By using equation (1.9) in (2.3) and differentiating logarithmically, we get

$$
\frac{1}{1-\gamma}\left(\frac{z\left(\mathscr{D}_{\lambda_{1}, \lambda_{2}}^{m, b}\left(a_{1}+1, b_{j}\right) f(z)\right)^{\prime}}{\mathscr{D}_{\lambda_{1}, \lambda_{2}}^{m, b}\left(a_{1}+1, b_{j}\right) f(z)}-\gamma\right)=p(z)+\frac{z p^{\prime}(z)}{(1-\gamma) p(z)+\left(a_{1}-1\right)+\gamma},
$$


since

$$
\frac{1}{1-\gamma}\left(\frac{z\left(\mathscr{D}_{\lambda_{1}, \lambda_{2}}^{m, b}\left(a_{1}+1, b_{j}\right) f(z)\right)^{\prime}}{\mathscr{D}_{\lambda_{1}, \lambda_{2}}^{m, b}\left(a_{1}+1, b_{j}\right) f(z)}-\gamma\right) \prec \phi(z),
$$

then applying Lemma 2.1, it follows that $p<\phi$. Hence the required result is obtained.

Taking $\phi(z)=(1+A z) /(1+B z),(-1 \leq B<A \leq 1)$ in Theorem 2.2 and in Theorem 2.3 we have:

Corollary 2.4. For $\left.\lambda_{2} \geq \lambda_{1} \geq 0, m, b \in \mathbb{N}_{0}, a_{i} \in \mathbb{C}, b_{j} \in \mathbb{C} \backslash\{0,-1,-2, \ldots\}\right),(i$ $=1, \ldots, r, j=1, \ldots, s, r \leq s+1 ; r, s \in \mathbb{N}_{0}$ and $0 \leq \gamma<1$ and $\Re\left\{a_{i}\right\}>1-\gamma$, we have

$$
\mathscr{Q}_{\lambda_{1}, \lambda_{2}}^{m+1, b}\left(a_{i}, b_{j} ; \gamma ; A, B\right) \subset \mathscr{Q}_{\lambda_{1}, \lambda_{2}}^{m, b}\left(a_{i}, b_{j} ; \gamma ; A, B\right),
$$

and

$$
\mathscr{Q}_{\lambda_{1}, \lambda_{2}}^{m, b}\left(a_{1}+1, b_{j} ; \gamma ; A, B\right) \subset \mathscr{Q}_{\lambda_{1}, \lambda_{2}}^{m, b}\left(a_{1}, b_{j} ; \gamma ; A, B\right) .
$$

Also, by taking $\phi(z)=((1+z) /(1-z))^{\beta},(0<\beta \leq 1)$ in Theorem 2.2 and in Theorem 2.3 we have:

Corollary 2.5. For $\left.\lambda_{2} \geq \lambda_{1} \geq 0, m, b \in \mathbb{N}_{0}, a_{i} \in \mathbb{C}, b_{j} \in \mathbb{C} \backslash\{0,-1,-2, \ldots\}\right),(i=1, \ldots, r, j=1, \ldots, s$, $r \leq s+1 ; r, s \in \mathbb{N}_{0}$ and $0 \leq \gamma<1$ and $\Re\left\{a_{i}\right\}>1-\beta$, we have

$$
\mathscr{S}_{\lambda_{1}, \lambda_{2}}^{m+1, b}\left(a_{i}, b_{j} ; \gamma, \beta\right) \subset \mathscr{S}_{\lambda_{1}, \lambda_{2}}^{m, b}\left(a_{i}, b_{j} ; \gamma, \beta\right),
$$

and

$$
\mathscr{S}_{\lambda_{1}, \lambda_{2}}^{m, b}\left(a_{1}+1, b_{j} ; \gamma ; \beta\right) \subset \mathscr{S}_{\lambda_{1}, \lambda_{2}}^{m, b}\left(a_{1}, b_{j} ; \gamma, \beta\right) .
$$

Corollary 2.6. For $\left.\lambda_{2} \geq \lambda_{1} \geq 0, m, b \in \mathbb{N}_{0}, a_{i} \in \mathbb{C}, b_{j} \in \mathbb{C} \backslash\{0,-1,-2, \ldots\}\right),(i=1, \ldots, r, j=1, \ldots, s$, $r \leq s+1 ; r, s \in \mathbb{N}_{0}, 0 \leq \gamma<1$ and $\Re\left\{a_{i}\right\}>1-\beta$, we have

$$
\mathscr{C}_{\lambda_{1}, \lambda_{2}}^{m+1, b}\left(a_{i}, b_{j} ; \gamma, \beta\right) \subset \mathscr{C}_{\lambda_{1}, \lambda_{2}}^{m, b}\left(a_{i}, b_{j} ; \gamma, \beta\right),
$$

and

$$
\mathscr{C}_{\lambda_{1}, \lambda_{2}}^{m, b}\left(a_{1}+1, b_{j} ; \gamma ; \beta\right) \subset \mathscr{C}_{\lambda_{1}, \lambda_{2}}^{m, b}\left(a_{1}, b_{j} ; \gamma, \beta\right) .
$$

Proof. We will proof the first relation and by the same method we can proof the second relation

$$
\begin{aligned}
f(z) \in \mathscr{C}_{\lambda_{1}, \lambda_{2}}^{m+1, b}\left(a_{i}, b_{j} ; \gamma, \beta\right) & \Leftrightarrow z f^{\prime}(z) \in \mathscr{S}_{\lambda_{1}, \lambda_{2}}^{m+1, b}\left(a_{i}, b_{j} ; \gamma, \beta\right) \\
& \Leftrightarrow z f^{\prime}(z) \in \mathscr{S}_{\lambda_{1}, \lambda_{2}}^{m, b}\left(a_{i}, b_{j} ; \gamma, \beta\right) \\
& \Leftrightarrow \mathscr{D}_{\lambda_{1}, \lambda_{2}}^{m, b}\left(a_{i}, b_{j}\right)\left(z f^{\prime}(z)\right) \in S^{*}(\gamma, \beta) \\
& \Leftrightarrow z\left(\mathscr{D}_{\lambda_{1}, \lambda_{2}}^{m, b}\left(a_{i}, b_{j}\right) f(z)\right)^{\prime} \in S^{*}(\gamma, \beta) \\
& \Leftrightarrow \mathscr{D}_{\lambda_{1}, \lambda_{2}}^{m, b}\left(a_{i}, b_{j}\right) f(z) \in C(\gamma, \beta) \\
& \Leftrightarrow f(z) \in \mathscr{C}_{\lambda_{1}, \lambda_{2}}^{m, b}\left(a_{i}, b_{j} ; \gamma, \beta\right) .
\end{aligned}
$$


Theorem 2.7. Let $\phi(z)$ be convex univalent in $\mathbb{U}$ with $\phi(0)=1$ and $\Re\{\phi(z)\} \geq 0$. Iff $\in \mathscr{A}$ satisfies the condition

$$
\frac{1}{1-\gamma}\left(\frac{z\left(\mathscr{D}_{\lambda_{1}, \lambda_{2}}^{m, b}\left(a_{i}, b_{j}\right) f(z)\right)^{\prime}}{\mathscr{D}_{\lambda_{1}, \lambda_{2}}^{m, b}\left(a_{i}, b_{j}\right) f(z)}-\gamma\right) \prec \phi(z) \quad(0 \leq \gamma<1 ; z \in \mathbb{U})
$$

then

$$
\frac{1}{1-\gamma}\left(\frac{z\left(\mathscr{D}_{\lambda_{1}, \lambda_{2}}^{m, b}\left(a_{i}, b_{j}\right) F_{c}(z)\right)^{\prime}}{\mathscr{D}_{\lambda_{1}, \lambda_{2}}^{m, b}\left(a_{i}, b_{j}\right) F_{c}(z)}-\gamma\right) \prec \phi(z) \quad(0 \leq \gamma<1 ; z \in \mathbb{U}),
$$

where $F_{c}$ be the generalised Bernardi-Libera-Livington integral operator defined by [11]-[13].

$$
\begin{aligned}
F_{c}(z)=\frac{c+1}{z^{c}} \int_{0}^{z} t^{c-1} f(t) d t \quad(c>-1), & \\
& =z+\sum_{n=2}^{\infty} \frac{c+1}{n+c} a_{n} z^{n} .
\end{aligned}
$$

Proof. From (2.5), we have

$$
z\left(\mathscr{D}_{\lambda_{1}, \lambda_{2}}^{m, b}\left(a_{i}, b_{j}\right) F_{c}(z)\right)^{\prime}=(c+1) \mathscr{D}_{\lambda_{1}, \lambda_{2}}^{m, b}\left(a_{i}, b_{j}\right) f(z)-c \mathscr{D}_{\lambda_{1}, \lambda_{2}}^{m, b}\left(a_{i}, b_{j}\right) F_{c}(z) .
$$

Now, let

$$
p(z)=\frac{1}{1-\gamma}\left(\frac{z\left(\mathscr{D}_{\lambda_{1}, \lambda_{2}}^{m, b}\left(a_{i}, b_{j}\right) F_{c}(z)\right)^{\prime}}{\mathscr{D}_{\lambda_{1}, \lambda_{2}}^{m, b}\left(a_{i}, b_{j}\right) F_{c}(z)}-\gamma\right),
$$

where $p \in \mathscr{P}$. Then by using (2.6) in (2.7), we obtain

$$
(1-\gamma) p(z)+c+\gamma=\frac{(c+1) \mathscr{D}_{\lambda_{1}, \lambda_{2}}^{m, b}\left(a_{i}, b_{j}\right) f(z)}{\mathscr{D}_{\lambda_{1}, \lambda_{2}}^{m, b}\left(a_{i}, b_{j}\right) F_{c}(z)} .
$$

Differentiating both sides of (2.8) logarithmically, we get

$$
p(z)+\frac{z p^{\prime}(z)}{c+\gamma+(1-\gamma) p(z)}=\frac{1}{1-\gamma}\left(\frac{z\left(\mathscr{D}_{\lambda_{1}, \lambda_{2}}^{m, b}\left(a_{i}, b_{j}\right) f(z)\right)^{\prime}}{\mathscr{D}_{\lambda_{1}, \lambda_{2}}^{m, b}\left(a_{i}, b_{j}\right) f(z)}-\gamma\right),
$$

since

$$
\frac{1}{1-\gamma}\left(\frac{z\left(\mathscr{D}_{\lambda_{1}, \lambda_{2}}^{m, b}\left(a_{i}, b_{j}\right) f(z)\right)^{\prime}}{\mathscr{D}_{\lambda_{1}, \lambda_{2}}^{m, b}\left(a_{i}, b_{j}\right) f(z)}-\gamma\right) \prec \phi(z),
$$

then applying Lemma 2.1, it follows that $p<\phi$. Hence the required result is obtained.

Now, by letting $\phi(z)=(1+A z) /(1+B z),(-1 \leq B<A)$ in Theorem 2.4 , we have

Corollary 2.8. For $\left.\lambda_{2} \geq \lambda_{1} \geq 0, m, b \in \mathbb{N}_{0}, a_{i} \in \mathbb{C}, b_{j} \in \mathbb{C} \backslash\{0,-1,-2, \ldots\}\right),(i$ $=1, \ldots, r, j=1, \ldots, s, r \leq s+1 ; r, s \in \mathbb{N}_{0}, c>-\gamma$ and $0 \leq \gamma<1$. If $f \in \mathscr{Q}_{\lambda_{1}, \lambda_{2}}^{m, b}\left(a_{i}, b_{j} ; \gamma ; A, B\right)$, then $F_{c} \in \mathscr{Q}_{\lambda_{1}, \lambda_{2}}^{m, b}\left(a_{i}, b_{j} ; \gamma ; A, B\right)$, where $F_{c}$ given by (2.5). 
Also, by taking $\phi(z)=((1+z) /(1-z))^{\beta},(0<\beta \leq 1)$ in Theorem 2.4, we have

Corollary 2.9. For $\left.\lambda_{2} \geq \lambda_{1} \geq 0, m, b \in \mathbb{N}_{0}, a_{i} \in \mathbb{C}, b_{j} \in \mathbb{C} \backslash\{0,-1,-2, \ldots\}\right),(i=1, \ldots, r, j=1, \ldots, s$, $r \leq s+1 ; r, s \in \mathbb{N}_{0}, c>-\beta, 0<\beta \leq 1$ and $0 \leq \gamma<1$. If $f \in \mathscr{S}_{\lambda_{1}, \lambda_{2}}^{m, b}\left(a_{i}, b_{j} ; \gamma, \beta\right)$, then $F_{c} \in \mathscr{S}_{\lambda_{1}, \lambda_{2}}^{m, b}\left(a_{i}\right.$, $\left.b_{j} ; \gamma, \beta\right)$ where $F_{c}$ given by (2.5).

Corollary 2.10. For $\left.\lambda_{2} \geq \lambda_{1} \geq 0, m, b \in \mathbb{N}_{0}, a_{i} \in \mathbb{C}, b_{j} \in \mathbb{C} \backslash\{0,-1,-2, \ldots\}\right),(i=1, \ldots, r, j=1, \ldots, s$, $r \leq s+1 ; r, s \in \mathbb{N}_{0}, c>-\beta, 0<\beta \leq 1$ and $0 \leq \gamma<1$. If $f \in \mathscr{C}_{\lambda_{1}, \lambda_{2}}^{m, b}\left(a_{i}, b_{j} ; \gamma, \beta\right)$, then $F_{c} \in \mathscr{C}_{\lambda_{1}, \lambda_{2}}^{m, b}\left(a_{i}\right.$, $\left.b_{j} ; \gamma, \beta\right)$, where $F_{c}$ given by (2.5).

Proof. Let

$$
\begin{aligned}
f(z) \in \mathscr{C}_{\lambda_{1}, \lambda_{2}}^{m, b}\left(a_{i}, b_{j} ; \gamma, \beta\right) & \Leftrightarrow z f^{\prime}(z) \in \mathscr{S}_{\lambda_{1}, \lambda_{2}}^{m, b}\left(a_{i}, b_{j} ; \gamma, \beta\right) \\
& \Leftrightarrow F_{c}\left(z f^{\prime}(z)\right) \in \mathscr{S}_{\lambda_{1}, \lambda_{2}}^{m, b}\left(a_{i}, b_{j} ; \gamma, \beta\right) \\
& \Leftrightarrow z\left(F_{c}(z)\right)^{\prime} \in \mathscr{S}_{\lambda_{1}, \lambda_{2}}^{m, b}\left(a_{i}, b_{j} ; \gamma, \beta\right) \\
& \Leftrightarrow F_{c}(z) \in \mathscr{C}_{\lambda_{1}, \lambda_{2}}^{m, b}\left(a_{i}, b_{j} ; \gamma, \beta\right) .
\end{aligned}
$$

Note: Some other work related to differential operators and hypergeometric functions can be found in [14]-[16].

\section{Acknowledgements}

The work presented here was partially supported by FRGSTOPDOWN/2013/ST06/UKM/ $01 / 1$. The authors also would like to thank the referee for the comments on the article.

\section{References}

[1] J. Dziok and H. M. Srivastava, Classes of analytic functions associated with the generalized hypergeometric function, Applied Mathematics and Computation, 103(1999), 1-13.

[2] C. Selvaraj and K. R. Karthikeyan, Univalence of a general integral operator associated with the generalized hypergeometric function, Tamsui Oxford Journal of Mathematical Sciences, 26(2010), 41-51.

[3] J. E. Hohlov, Operators and operations on the class of univalent functions, Izv. Vyssh. Uchebn. Zaved. Mat., 10(197) (1978), 83-89.

[4] G. S. Salagean, Subclasses of univalent functions, Complex analysis-fifth Romanian-Finnish seminar, Part 1 (Bucharest, 1981), 362-372, Lecture Notes in Math., 1013, Springer, Berlin.

[5] F. M. Al-Oboudi, On univalent functions defined by a generalized Salagean operator, Int. J. Math. Math. Sci. 2004, no. 25-28, 1429-1436.

[6] S. Ruscheweyh, New criteria for univalent functions, Proceedings of the American Mathematical Society, 49(1975), 109-115.

[7] E. El-Yagubi and M. Darus, Subclasses of analytic functions defined by new generalised derivative operator, Journal of Quality Measurement and Analysis, 9(2013), 47-56.

[8] B. C. Carlson and D. B. Shaffer, Starlike and prestarlike hypergeometric functions, SIAM Journal on Mathematical Analysis, 15(1984), 737-745. 
[9] A. Cátás, On certain class of p-valent functions defined by a new multiplier transformations, Proceedings Book of the International Symposium G. F. T. A., 241-250, Istanbul Kultur University, Istanbul, Turkey, 2007.

[10] P. Eenigenburg, S. S. Miller, P. T. Mocanu, and M. O. Reade, On a Briot-Bouquet differential subordination, General Inequalities, vol. 64 of Internationale Schriftenreihe zur Numerischen Mathematik, 339-348, Birkhäuser, Basel, Switzerland, 1983.

[11] S. D. Bernardi, Convex and starlike univalent functions, Transactions of the American Mathematical Society, 135(1969), 429-446.

[12] R. J. Libera, Some classes of regular univalent functions, Proceedings of the American Mathematical Society, 16(1965), 755-758.

[13] A. E. Livington, On the radius of univalence of certain analytic functions, Proceedings of the American Mathematical Society, 17(1966), 352-357.

[14] F. Ghanim and M. Darus, A new class of meromorphically analytic functions with applications to the generalized hypergeometric functions, Abstract and Applied Analysis, Volume 2011 (2011), Article ID 159405, 10 pages.

[15] H. Aldweby and M. Darus, On harmonic meromorphic functions associated with basic hypergeometric functions, The World Scientific Journal: Mathematical Analysis, Volume 2013 (2013), Art. 164287, 7 pages.

[16] A. Mohammed and M. Darus, A generalized operator involving the q-hypergeometric function, Matematicki Vesnik 65(2013), 454-465.

School of Mathematical Sciences, Faculty of Science and Technology, Universiti Kebangsaan Malaysia, Bangi 43600 Selangor D. Ehsan, Malaysia.

E-mail: entisar_e1980@yahoo.com

School of Mathematical Sciences, Faculty of Science and Technology, Universiti Kebangsaan Malaysia, Bangi 43600 Selangor D. Ehsan, Malaysia.

E-mail: maslina@ukm.edu.my 